\title{
Natural Flow Convection in a vertically multilayered porous media with varying permiabilities (Part 1: Temperature and Flow fields)
}

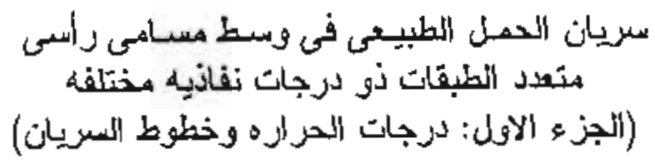

M. S. EL KADY

Mechanical Engineering Department

Mansoura University, Egypt

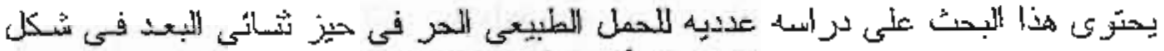

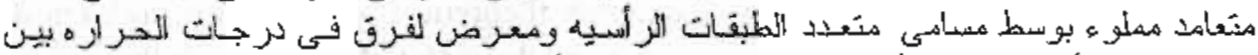

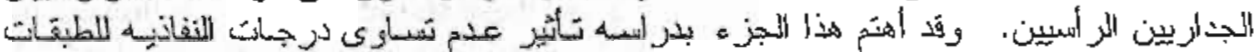

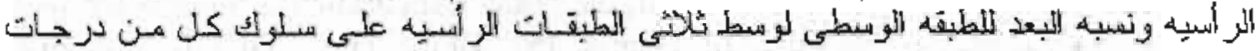

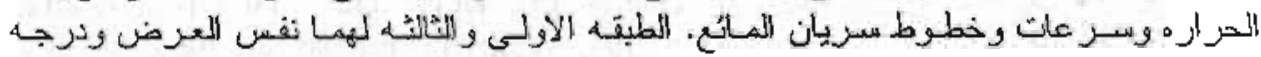

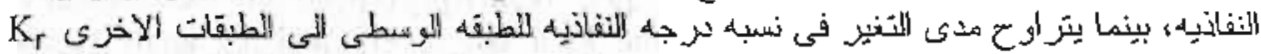

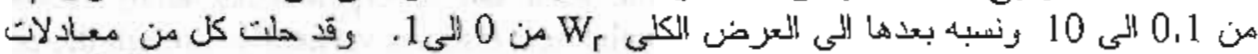

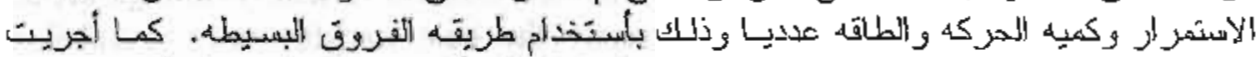

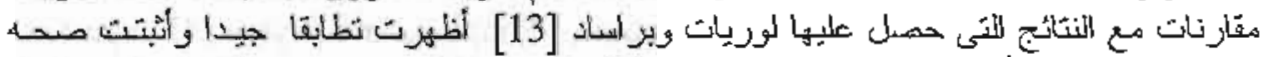

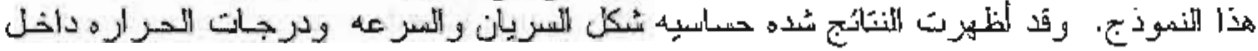
الوسط الثسمسأنى اللتغير في درجه النفانيه.

\section{ABSTRACT}

Convective heat transfer through a vertical multilayered porous media is examined numerically. The examination is focused upon the effect of the nonuniform permeabilities of the sublayers, with verical isothermal walls at different temperatures and horizontal perfect insulated walls. The study was done for a 3 layered porous medium in which the first and third layers have equal thicknesses and permeabilities, for a range of the permeability ratio $K_{r}$ of the core region from 0.1 to 10 and a range of the width ratio of the inner stib-layer to the total width $\left(W_{r}\right)$ varies form 0.0 to 1.0 . This part is concemed with the effect of the variable permeabilty on the temperature and flow fields and the second part of this work [14], is concerned with the effect of the variable permeability 
porous layers on the heat transfer. The governing equations have been written in terms of nondimensional variables and were solved using the finite difference method. A comparison is made with the results obtained by Lauriat and Prasad [13]. The comparison shows a very good agreement for the present results and hence proves the validity of the model. The results give a very good idea about the effect of the inhomogenity of the porous medium on the flow structure and temperatures.

\section{INTRODUCTION}

The heat transfer by natural convection across a porous medium heated from one side is a topic of fundamental importance in diverse fields such as thermal insulation engineering, geothermal reservior dynamics and grain storage. The basic model used so far in the study of porous media heated from the sides consists of a 2-dimensional layer with vertical isothermal walls at different temperatures and with adiabatic top and bottom walls. This model has been investigated extensively during the past 20 years. The first study of the problem, an experimental one, was reported by Schneider [1]. He investigated the natural convection heat transfer through granular material under the condition of fixed height and width. The first extensive theoretical work on this problem was performed by Chan et al. [2]. Later on, these studies were followed by a series of investigations, out of which the notable theoretical solutions by Weber [3], Bums et al. [4] walker and Homsy [5], Bejan and Tien [6], Bejan [7], Simpkins and Blythe [8], Blythe et al. [9]. Weber [3] reports an Oseen-linearized analysis of the boundary layer regime in a ta'l layer; Weber's analysis was improved by Bejan [7] to account for the heat transfer vertically through the core and, in this way, to predict correctly the heat transfer rates revealed by experiments and by numerical simulations. Simpkins and Blythe [8] reported an alternative theory for the boundary layer regime based on an original closed form solution, and for temperature dependent viscosity [9]. The corresponding flow in the shallow layer heated from the sides was documented by Walker and Homsy [5]. They developed an asymptotic solution for the flow and temperature fields inside a shallow layer using the aspect ratio as the small parameter. They showed that unlike the in the tall layers the core region plays an active role in the heat transfer processes. An approximate integral type solution for the same geometry was reported by Bejan and Tien [6]. Tong and Subramanian [10] have presented a boundary layer analysis for a Brinkman model, and have reporeted significant contributions of the diffusion term at high Rayleigh and Darcy numbers. Furthermore, Poulikakos and Bejan [11] and Prasad and Tuntomo [12] have considered the Forchheimer-extended Darcy model to study the inertia effects on free convection in a vertical cavity. Lauriat and Prasad [13] considered the 
Brinkman-extended Darcy equation of motion together with the transport tern and examined the significance of each term. Elkady [15] clarified numerically the effect of the dimensions of the rectangular cavity on the heat transfer through the inclined cavity with variable inclination angles from 0 to 180 .

The survied literature presented above have been concerned with natural convection in layers only filled with homogeneous porous medium, which may not be a good approximation for the porous layers in the real life. The purpose of this study is to analyse and understand the effect of the inhomogenity of the porous medium on the flow structure and the convective heat transfer in a vertical layered porous media with vertical isothermal walls at different temperatures. This part is concerned with the temperature and flow fjelds, and the effect of the variable permeability porous layers on the heat transfer is considered in the second part of this work [14].

\section{MATHEMATICAL MODEL AND SOLUTION PROCEDURE}

Consider a two-dimensional rectangular vertical cavity (shown in Fig. 1) of width $\mathrm{W}$ and height $\mathrm{H}$, filled with mtilayered porous medium. Each layer is homogenuous, isotropioc and has constant permeability $\mathrm{K}$. The porous medium is saturated with a single phase fluid of density $\rho$ and viscosity $\mu$. In Fig. $1 T_{H}$ and $T_{C}$ represent the hot and cold vertical walls of the cavity respectively while the horizontal top and bottom walls are insulated. The effect of both the drag and inertia are neglected, and the flow will obey Darcy's law .

The fluid properties are assumed to be constant except for the density change with temperature which gives rise to the buoyancy force, this is treated by invoking the Boussinesq approximation. While the permeability values $\mathrm{K}$ of the porous layers are different, the values of the thermal diffusivity in the layers are the same. This assumption is done to study the effects of the change of the permeability alone. With these assumptions, the conservation equations for the mass, rnomentum and energy for each layer become:

Continuity

Momentum (Darcy's law)

$$
\partial u / \partial x+\partial v / \partial y \quad=0
$$

$$
\begin{aligned}
& \partial p / \partial x+(\mu / K) u=0 \\
& \partial p / \partial y+(\mu / K) v=\rho g \beta\left(T-T_{C}\right)
\end{aligned}
$$

Energy

$$
\mathrm{u} \partial \mathrm{T} / \partial \mathrm{x}+\mathrm{v} \partial \mathrm{T} / \partial \mathrm{y}=\alpha\left(\partial^{2} \mathrm{~T} / \partial \mathrm{x}^{2}+\partial^{2} \mathrm{~T} / \partial \mathrm{y}^{2}\right)
$$


where $u, v, p, \alpha, \beta, g, \mu$ and $K$ are the velocity components in the $x$ and $y$ directions, pressure, thermal diffusivity, coefficient of thermal expansion, acceleration due to gravity, Kinematic viscosity and Permeability of the porous media, respectively.

Eliminating the pressure terms from equations (2) and (3), introducing the stream function $\varphi$ as $u=d \varphi / d y$ and $v=-d \varphi / d x$, and scalling all the variables by appropriate characteristic values of those variables defined by:

$$
\mathrm{X}=\mathrm{x} / \mathrm{W}, \quad \mathrm{X}=\mathrm{y} / \mathrm{H}, \Psi=\varphi \mathrm{H} / \propto \mathrm{W} \text {, and } \theta=\left(\mathrm{T}-\mathrm{T}_{\mathrm{C}}\right) /\left(\mathrm{T}_{\mathrm{H}}-\mathrm{T}_{\mathrm{C}}\right)
$$

the governing equations (1)-(4) can be transformed into a non-dimensional stream function and a temperature equations

$$
\begin{gathered}
\partial^{2} \Psi / \partial \mathrm{X}^{2}+\left(1 / \mathrm{A}^{2}\right) \partial^{2} \Psi / \partial \mathrm{Y}^{2}=-\operatorname{Ra}(\partial \theta / \partial \mathrm{X}) \\
\mathrm{U} \partial \theta / \partial \mathrm{X} \div \mathrm{V} \partial \theta / \partial \mathrm{Y}=\mathrm{A}^{2} \cdot \partial^{2} \theta / \partial \mathrm{X}^{2}+\partial^{2} \theta / \partial \mathrm{Y}^{2}
\end{gathered}
$$

where $\mathrm{A}$ is the aspect ratio of the cavity $=\mathrm{H} / \mathrm{W}$ and $\mathrm{Ra}$ is the Darcy-Rayliegh number based on the height of the cavity $\mathrm{H}$, and is given by :

$$
\mathrm{Ra}=\mathrm{Kg} \beta\left(\mathrm{T}_{\mathrm{H}}-\mathrm{T}_{\mathrm{C}}\right) \mathrm{H} / \alpha \mathrm{v}
$$

Because the vertical layers have different permeiabilities $K$, the DarcyRayleigh number $\mathrm{Ra}$ will be different for each layer. Taking the permeability ratio for each layer as $K_{r}=K / K_{H}$, the Darcy-Rayleigh number for each layer will be: $\mathrm{Ra}=\mathrm{K}_{\mathrm{r}} \cdot \mathrm{Ra}_{\mathrm{H}}$

where $K_{H}$, Ra $a_{H}$ are taken as the permiability value and the Darcy-Rayleigh number in the layer in contact with the hot wall.

On the solution, the boundary conditions for the nondimensional stream function and temperature are:

$$
\begin{aligned}
& \Psi=0 \text { on all walls, } \quad \partial \theta / \partial Y=0 \quad \text { for } Y=0,1 \text { and } 0<X<1, \\
& \theta=1 \text { for } X=0 \text { and } 0<Y<1, \quad \theta=0 \text { for } X=1 \text { and } 0<Y<1
\end{aligned}
$$

The solution of the governing differential equations (5) and (6) has been obtained numerically by using the finite difference scheme presented by Patankar [18]. The solution consists of the stream function and the temperature fields as well as the velocities in the $\mathrm{x}$ and $\mathrm{y}$ directions. More detailed information about the numerical procedure can be found in [16]. 


\section{RESULTS AND DISCUSSION}

The two dimensional natural convection in a mulalayered with different permeabilities fluid saturated porous medium has been analyzed for vertical isothermal walls at different temperatures and with adiabatic top and bottom walls. The study is made for 3 layered porous medium in which the first and third layers have equal thicknesses and permeabilities. A wide range of the effective parameters are considered. The penneability Darcy Rayleigh number $\mathrm{Ra}$ up to 6000 . The effect of these parameters on the temperature, streamlines and the velocity of the saturated fluid flow inside the porous medium are studied.

\subsection{The validity of the model}

In the following study in sections 3.2 and 3.3 , the cases of $W_{r}=0.0$ and 1.0, correspond to those cases of a uniformly filled cavity, and the results obrained here for these cases are in good agreement with those obtained by the author in previous study [15]. Another comparison is done with the results obtained by Lauriat and Prasad [13] for a case of a single layer porous media with $W_{r}=0, A=5$ and $R a=250,1000$ and 2500 . The values of the vertical velocity $\mathrm{V} / \mathrm{Ra}$ and the temperature $\theta$ at the midheight section where $Y=0.5$ and the horizontal velocity $\mathrm{U} / \mathrm{Ra}$ at $\mathrm{X}=0.5$ are translated into the corresponding notations and expressions by this work and compared with it. The comparison which is shown in Figs 2-3 shows a very good agreement and proves the validity of the model.

\subsection{Effect of sublayers width}

Figs. 4-10 show the effect of the width ratio of the inner sublayer $W_{r}$ on the streamlines and isothermal lines. The width ratio $W_{r}$ takes the values 0.0 , $0.2,0.4,0.6,0.8,1.0$.

Figs 4-7 show the case where the penneability of the inner layer is five times greater than the outer layers $K_{r}=5$, the aspect ratio $A=3$ and $R a=150$. In this case of $\mathrm{W}_{\mathrm{r}}=0$, where the whole cavity is filled with low permeability porous media, the streamlines shown in Fig.4, tend to be in the outer region of the sublayers with less flow in the core. With the appearance of the higher permeability inner sublayer, an attraction appears for the flow towards the core, and the tendency for more flow to be initiated in this attractive layer exists. With the increase of $\mathrm{W}_{\mathrm{r}}$ the attraction for the flow towards the core increases, and higher values of streamfunction exists also, carrying more convective heat 
from the hotter side to the colder one. The flow rate increases with the increase of $W_{r}$ and the slope of the axis of the cells changes significantly. For example at $W_{r}=0$, the axis is close to the vertical middle plane, but with the increase of $W_{r}$, the axis moves towards the diagonal of the cavity. Therefore, the maximum velocities (horizontal and vertical components) drift from the vertical and horizontal middle planes to the corners (left bottom and right top corners).

An indication for the transport of energy by convection between the two isothermal vertical walls is the rate of flow inside the cavity, which can be expressed by the maximum value of the streamfunction and the mean velocity of the flow. To express these two functions two variables are used in [15-17], and are defined as:

$$
\Psi_{\max }= \pm \max |\Psi(x, y)| \text { and } U_{m}={ }_{A} \int\left(U^{2}+V^{2}\right) d A
$$

where $\Psi_{\max }$ is the maximum value of the non-dimensional streamfunction, $t$ are taken for counter clockwise and clockwise circulation respectively and $U_{m}$ is expressed as a function of the average fuid speed over the area $\mathrm{A}$.

Fig. 5 shows the behavior of those both functions expressed by $\mathrm{U}_{\mathrm{m}} / \mathrm{U}_{\mathrm{m} 0}$ and $\Psi_{\max } / \Psi_{\max 0}$ with the increase of $\mathrm{W}_{\mathrm{r}}$. Where $U_{\operatorname{mo}}$ and $\Psi_{\max 0}$ are the functions of the mean velocity and maximum streamfunction in the case of $\mathrm{W}_{\mathrm{r}}=0$. The two figures show a significant increase of both the two functions with the in rease of $W_{r}$ indicating the increase of the transport energy by convection part in the whole layers.

The behavior of the isothermal lines for different inner sublayer width ratios $\mathrm{W}_{\mathrm{r}}$ is shown in Fig. 6 . It is noteced that the interior temperature distribution is close to a straight line in the central part of the inner sublayer. The slope of these isothermal lines shows that the heat is transfered by both the conduction and convection. With the increase of the width of the attractive inner sublayer, the above modification in velocity field with the $\mathrm{W}_{\mathrm{r}}$ changes the isotherm pattens accordingly. The isothermal lines deviate in the inner sublayer towards the horizontal direction (normal to the walls of the heat flow) indicating the increase of the transport of energy by convection part in the core region. The temperature gradients near the bottom left and the right top corners are greatly modified to a sharp temperature gradients. Thus the transport of energy due to cross flow near the horizontal walls has, thus, increased also.

Fig. 7 shows the effect of the inner sublayer width ratio $W_{r}$ on both the temperature and velocity distributions at the middle height of the porous media 
$Y=0.5$ in the half of the width adjacent to the hotter side. For the case of $\mathrm{W}_{\mathrm{r}}=0.0$, where the whole cavity is filled with low permeability porous media, both the temperature and velocity distribution curves are nearly straight lines. With the increase of $W_{\mathrm{r}}$ both the temperature and velocity curves deviate from the straight line condition in the inner sublayer and remain taking the nearly straight line shape in the lower permeability outer layer near the botter side, with a significant increase in the velocity field and decreasing temperatures. The step variation in the permeability across the interface of the two sublayers induces step changes in the slopes of both velocities and temperatures. The break points in both fields are shown by the symbol (o).

Figs. 8-10 show another case, where the permeability of the inner layer is five times less than that in the outer layers, i.e. $K_{r}$ for the inner sublayer $=0.2$, the aspect ratio $A=3$ and $R a=400$. The behavior of the streamlines for different values of $\mathrm{W}_{r}$ is shown in Fig.8. For the case of uniform porous media where $\mathrm{W}_{\mathrm{r}}=0.0$, i.e. high permeability layer, the temperature difference across the two vertical walls causes a relatively high velocity flow all over the layer. As the less permeability inner sublayer appears, a new resistance appears for the flow in the core of the inner sublayer. With the increase of $W_{r}$, the damping for the existing flow in the core increaseses. The slope of the axis of the cells changes significantly. At $\mathrm{W}_{\mathrm{r}}=0$ the axis is close to the diagonal of the cavity, but with the increase of $W_{r}$, the axis moves towards the vertical middle plane indicating that the axirnum velocities (horizontal and vertical components) drift from the corners (left bottom and right top comers) to the vertical and horizontal middle planes. Therefore, the flow is attracted to be vertically in the outer layers, where the higher permeability exists, and is forced to be exchanged between the outer sublayers carrying the convective heat energy part through the upper and lower ends of the inner layer, and less flow of convective energy transfer through the core region.

Fig. 9 shows the behavior of the functions expressed by $U_{m} / U_{m o}$ and $\Psi_{\max } / \Psi_{\max 0}$ with the increase of $\mathrm{W}_{\mathrm{r}}$ where $\mathrm{U}_{\operatorname{mo}}$ and $\Psi_{\max 0}$ are the functions of the mean velocity and maximum streanfunction in the case of $W_{\mathrm{r}}=0.0$. The two figures show a significant decrease of both the two functions which indicates the decrease of both the mean velocity of the flow and the rate of flow inside the cavity, which in turns indicates the decrease of the transport energy by convection part in the whole layers.

Fig. 10 shows the behavior of the isothermal lines for different values of $W_{r}$. In the core of the inner sublayer, where, less flow exists, the above 
modification in the velocity field with the increase of $\mathrm{W}_{\mathrm{r}}$ changes the isotherm pattem accordingly. The isothermal lines deviate towards the vertical direction (parallel to the isothermal walls) indicating the decrease of convection heat flow through the core region. The sharp temperature gradients near the bottom left and the right top comers are greatly modified, and the transport of energy due to the cross flow near the horizontal walls has, thus, decreased.

\subsection{Effect of permeability ratios}

Figs. 11-13 show the streamlines and the isothermal lines behavior for three layers porous media with equal widths, $\mathrm{Ra}=250$. Aspect ratio $\mathrm{A}=3$ and different permeability ratios for the inner to the outer sublayers $K_{r}$ from 10 to 0.1 .

The behavior of the streamlines for different values of $K_{r}$ is shown in Fig.11. For the case of uniform porous media where $K_{r}=1$, i.e. high permeability one layer, the temperature difference across the two vertical walls causes a relatively high velocity flow all over the layer. For the case of $\mathrm{K}_{r}>1$, with the increase of $K_{r}$, the attraction for the outer streamlines to change its direction gradually towards the core of the inner sublayer increases, besides the increase of the chance for the fluid rate of flow in the core to increase, carrying more convective heat from the hotter side to the colder one. For the case of $K_{r}<1$, with the decrease of the permeability ratio $K_{r}$ the streamlines find an increased resistance to flow in the core of the inner sublayer, and is forced to change its Zirection gradually and compressed in the outer sublayers, tending to be parallel to the outer vertical walls. The flow is then forced to be exchanged between the outer sublayers through the upper and lower ends of the inner sublayer.

This phenomenon reflects itself on the maximum value of the streanfunction and the mean velocity of the flow in the cavity. Fig. 12 shows the increase of both $U_{m} / U_{m 1}$ and $\Psi_{\max } / \Psi_{\max 1}$ with the increase of the permeability ratio of the inner sublayer. where $U_{m 1}$ and $\Psi_{\max 1}$ are the functions of the mean velocity and maximum streamfunction in the case of $\mathrm{K}_{\mathrm{r}}=1$.

Fig. 13 shows the behavior of the isothermal lines for different values of $K_{r}$. With the decrease of the inner sublayer permeability ratio $K_{r}$ the above modification in the velocity field changes the isotherm pattern accordingly. The isothermal lines deviate towards the vertical direction (parallel to the isothermal walls) indicating the decrease of convection heat flow through the core region. The sharp temperature gradients near the bottom left and the right top corners are greatly modified, and the transport of energy due to the cross flow near the horizontal walls has, thus, decreased also. 


\section{CONCLUSIONS}

This paper outlined a numerical solution for the phenomenon of the furid flow by natural convection in a two dimensional vertical multilayered porous medium with different permeabilites and heated from the side. The study is focused on the effect of the non uniform permeability of the sublayers on the behavior of the tempreture and streamlines fields. The results indicate the following:

With the increase of $K_{r}$ or the increase of $W_{r}$ for $K_{r}>1$ or the decrease of $\mathrm{W}_{r}$ for $\mathrm{K}_{\mathrm{r}}<1$,

- the slope of the axis of the streamlines cells moves from the vertical middle plane towards the diagonal of the cavity.

- the maximum velocities (horizontal and vertical components) drift from the vertical and horizontal middle planes to the comers (left bottom and right top corners).

- the attraction for the flow towards the core increases, the flow rate and the mean velocity in the cavity increase and higher values of streamfunction exist, carrying more convective heat from the hotter side to the colder one.

- the interior temperature distribution is close to a straight line in the central part of the inner sublayer. The isothermal lines deviate from the vertical direction (parallel to the isothermal walls) towards the horizontal direction (normal to the isothermal walls) indicating the increase of the transport of energy by convection part in the core region.

- the temperature gradients near the bottom left and the right top corners are greatly modified to a sharp temperature gradjeuts, indicating the increase of the transport of energy due to cross flow near the horizontal walls.

With the decrease of $K_{r}$ or the increase of $W_{r}$ for $K_{r}<1$ or the decrease of $W_{r}$ for $K_{r}>1$, a damping for the existing flow in the core increases, and the tlow is attracted to be vertical in the outer layers, where the higher permeability exists, and is forced to be exchanged between the outer sublayers carnying the convective heat energy part through the upper and lower ends of the inner layer.

\section{NOMENCLATURE}

$\begin{array}{ll}\text { A } & \text { Aspect ratio }=\mathrm{H} / \mathrm{W} \\ \mathrm{g} & \text { Acceleration due to gravity, } \mathrm{m}^{2} / \mathrm{s} \\ \mathrm{H} & \text { Feight of the porous material, } \mathrm{m} \\ \mathrm{K} & \text { Permeability of the porous layer, } \mathrm{m}^{2} \\ \mathrm{~K}_{\mathrm{H}} & \text { Permeability of the porous layer adjacent to the hot wall, } \mathrm{m}^{2}\end{array}$


$\mathrm{K}_{\mathrm{r}} \quad$ Ratio of the permeability of the porous layer to the penreability of the porous layer adjacent to the hot wall $=\mathrm{K} / \mathrm{K}_{\mathrm{H}}$

p Pressure, $\mathrm{Pa}$

Ra Darcy-Rayleih number $=\mathrm{g} \beta \mathrm{KH}\left(\mathrm{T}_{\mathrm{H}}-\mathrm{T}_{\mathrm{C}}\right) / \alpha \mathrm{v}$

$R_{H} \quad$ Darcy-Rayleih number for the layer adjacent to the hot wall

$T \quad$ Temperature, $\mathrm{K}$

$T_{H}, T_{C} \quad$ Temperature of the hot and cold isothermal surfaces, $K$

$\mathrm{u}, \mathrm{r} \quad$ Field velocities in the $\mathrm{x}$ and $\mathrm{y}$ directions, $\mathrm{m} / \mathrm{s}$

$\mathrm{U}, \mathrm{V} \quad$ Non-dimensional field velocities in the $\mathrm{X}$ and $\mathrm{Y}$ directions respectively

$\mathrm{U}_{\mathrm{m}} \quad$ Fluid non-dimensional average velocity

$\mathrm{x}, \mathrm{y} \quad$ Spatial coordinates

$X Y \quad$ Dimensionless distances in the $\mathrm{x}$ and $\mathrm{y}$ axis respectively

W Width of the porous material, $m$

Wr Width ratio

$\alpha \quad$ Thermal diffusivity of the porous layers, $\mathrm{m}^{2} / \mathrm{s}$

$\beta \quad$ Coefficient of volumetric thermal expansion, $1 / K$

$\mu \quad$ Dynamic viscosity of the fluid

u Kinematic viscoisity of the fluid, $\mathrm{m}^{2} / \mathrm{s}$

$\rho \quad$ Fluid density

$\varphi \quad$ Stream function

$\Psi \quad$ Dimensionless stream function

$\Psi_{\max } \quad$ Maximum extreamum value of the strean function

$\theta \quad$ Non-dimensional temperature $=\left(\mathrm{T}-\mathrm{T}_{\mathrm{C}} /\left(\mathrm{T}_{\mathrm{H}}-\mathrm{T}_{\mathrm{C}}\right)\right.$

\section{REFERENCES}

1. Schneider, K.,J. "Investigation of the influence of free thermal convection on heat transfer through granular material," Proceedings, International Institute of Refrigeration, pp 247-253, 1963.

2. Chan, B. K.C., Ivey, C. M., and Barry, J. M.,"Natural convection in enclosed porous media with rectangular boundaries", ASME J. of Heat Transfer, Vol.2, pp 21-27, 1970

3. Weber, J. E.,"The boundry layer regime for convection in a vertical porous layer." Int. Journal of Heat and Mass Transfer, Vol. 18, pp 569-573, 1975.

4. Burns, P. J., Chow, L. C. and Tien. C. L. "Convection in a vertical slot filled with porous insulation". Int. Journal of Heat and Mass transfer. Vol. 20, pp919-929, 1977.

5. Walker, K. L. and Homsy, G.M.,"Convection in a porous cavity" Journal of Fluid Mechanics, Vol.87, pp 449-474, 1978. 
6. Bejan, A. and Tien, C.,"Natural convection in a horizontal porous medium subjected to an end to end temperature difference". J. of heat transfer, Vol. 100 , pp 19l-198, 1978.

7. Bejan, A., "On the boundry layer regime in avertical enclosure filled with porous medium". Heat Mass Transfer, Vol 6, pp 93-102, 1979

8. Simpkins, P., and Blythe, P., "Convection in a porous layer" Ent. Journal Heat Mass Transfer, Vol. 23, pp 881-887, 1980.

9. Blythe, P. A., and Simpkins, P. G.,"Convection in a porous layer for a temperature dependent viscosity", Int. Joumal Heat Mass Transfer, Vol. 24, pp 497-506, 1981.

10. Tong, $T$, and Subramanian, $E$., "A boundary layer analysis for natural convection in a porous enclosure-use of the Brinkman extended Darcy model". Int. Joumal of Mass and Heat Transfer, Vol.28, pp 563-5\%1, 1985.

11. Poulikakos, D., and Bejan, A., "The Departure from Darcy Flow in Natural Convection in a Vertical Porous Layer", Physics of Fluids, vol. 28, pp. 3477$484,1985$.

12. Prasad, V., and Tuntoma, A., "Inertia effect on natural convection in a vertical Porous cavity", Numerical Heat Transfer, 1987

13. Lauriat, G. and Prasad, V., "Natural convection in a vertical porous cavity: a numerical study for Brinkman-extended Darcy formulation", Trans.of the ASME, Vol 109, pp 688-696, 1987.

14. El Kady, M.S.,"Natural flow convection in a vertical multi- layered porous media with varying permeabilities (Part 2:Heat transfer) to be published in Mansoura Engineering Joumal.

15. E1 Kady, M.S.,"Natural convection in an inclined rectangular porous medium at various angles and aspect ratios", Mansoura Engineering Joumal (MEJ), vol 15, No. 2, pp M50-M68, 1990.

16. El Kady, M.S.,"Numerical study of natural convection in a rectangular porous medium with vertical temperature gradient", Mansoura. Eng. Journal (MEJ), vol. 15, No. 1, pp M72-M87, 1990.

17. El Kady, M.S.,"Effect of the Aspect ratio on the natural convection in a rectangular porous medium" Mansoura Engineering Journal (MEJ), vol 15, No. 1, pp M95-M107, 1990.

18. Patankar, S., "Numerical Heat transfer and fluid flow" Mc Graw Hill, New York, 1980. 


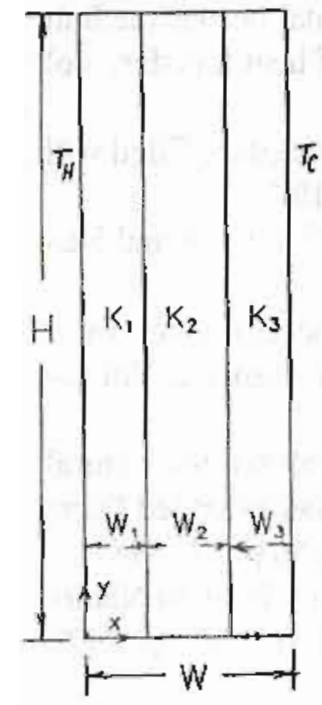

Fig. 1 Schematic diagram of the rectangular multilayered porous cavity

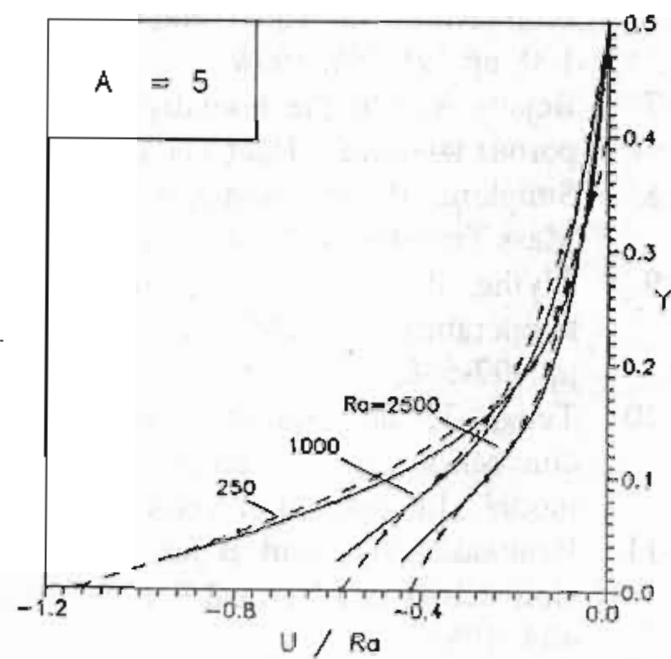

Fig. 3 The horizontal velocity U/Ra at the middle vertical plane $X=0.5$ ..... Lauriat and Prasad [13], Present work
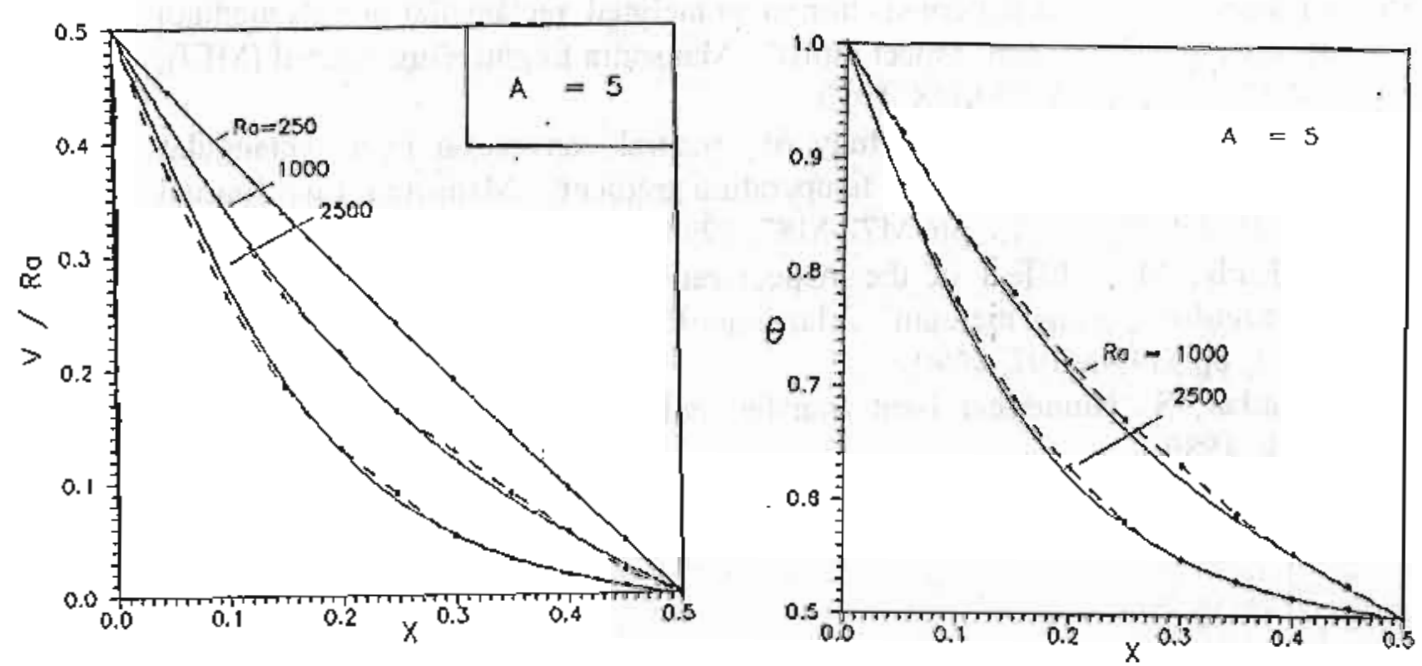

Fig. 2 The Vertical velocity V/Ra and the non-dimensional temperature $\theta$ at the midheight section where $X=0.5$

. . . - Lauriat and Prasad [13],

Present work 

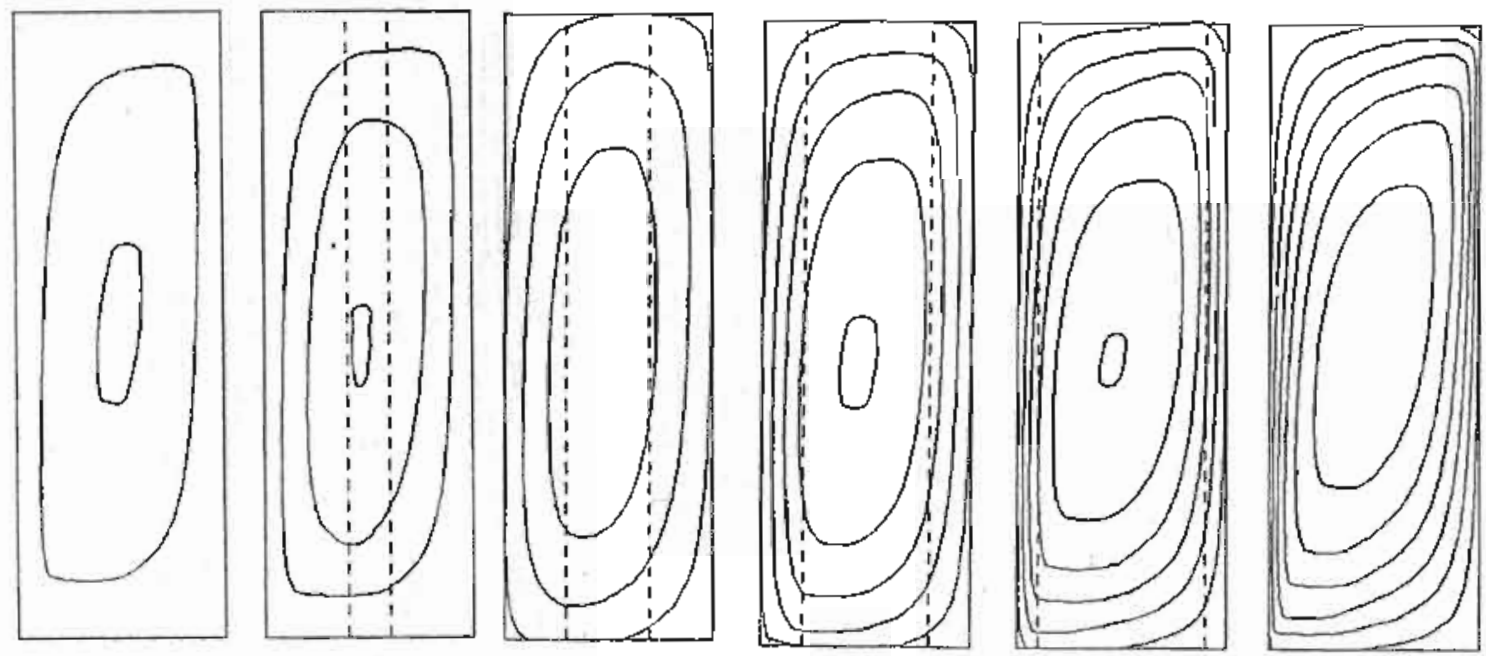

Fig. 4 Streamlines for $A=3, \quad K_{r}=5$ and $R a=150$

$$
\left(\Psi_{1}=8, \Delta \Psi=8\right)
$$
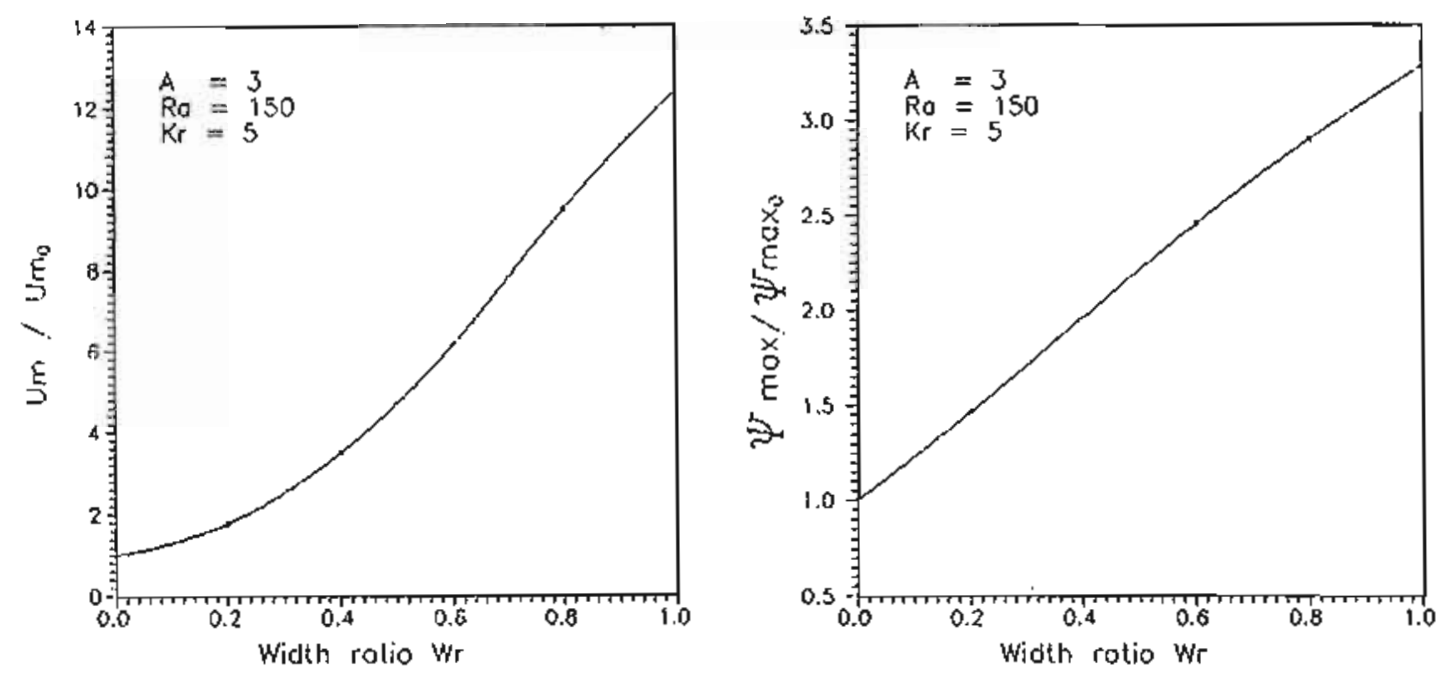

Fig. 5 Variation of the nondimenstonal average velocity and Streamfunction with the width ratio of the inner sublayer for $A=3, K_{r}=5$ and $R a=150$ 

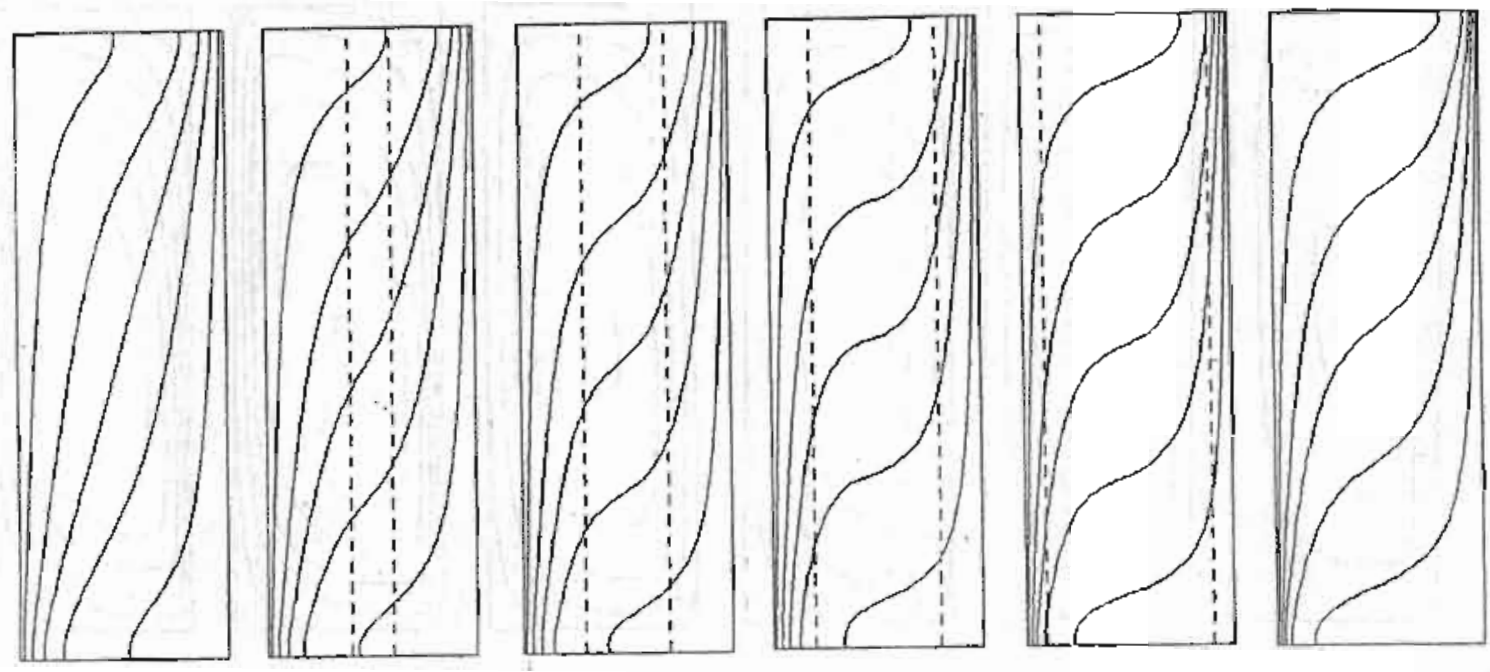

Fig. 6 isotherms for $A=3, K_{r}=5$ and $R a=150$

$$
\left(\theta_{1}=0.1, \quad \Delta \theta=0.2\right)
$$
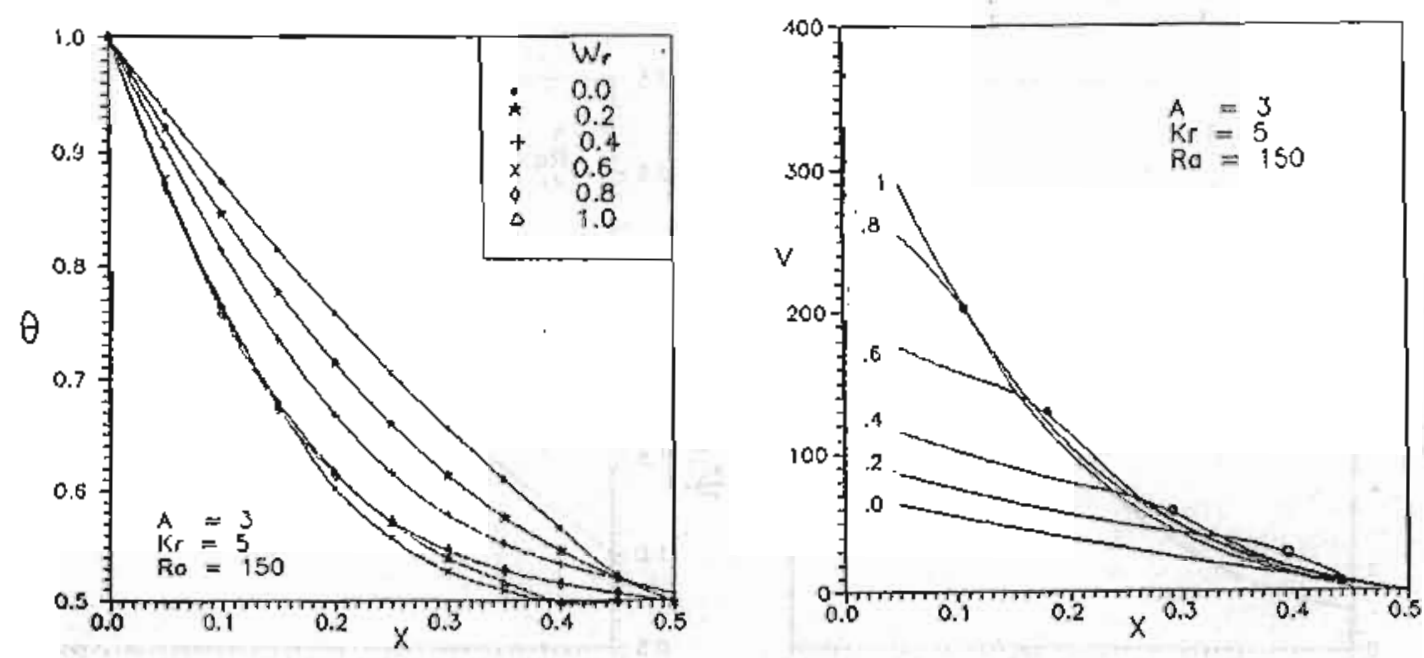

Fig. 7 The effect of the imer sublayer width ratio $\mathrm{W}_{\mathrm{r}}$ on the temperature and velocity distributions at the midheight section where $\mathrm{Y}=0,5$ for $\mathrm{A}=3, \mathrm{~K}_{\mathrm{r}}=5$ and $\mathrm{Ra}=150$. 

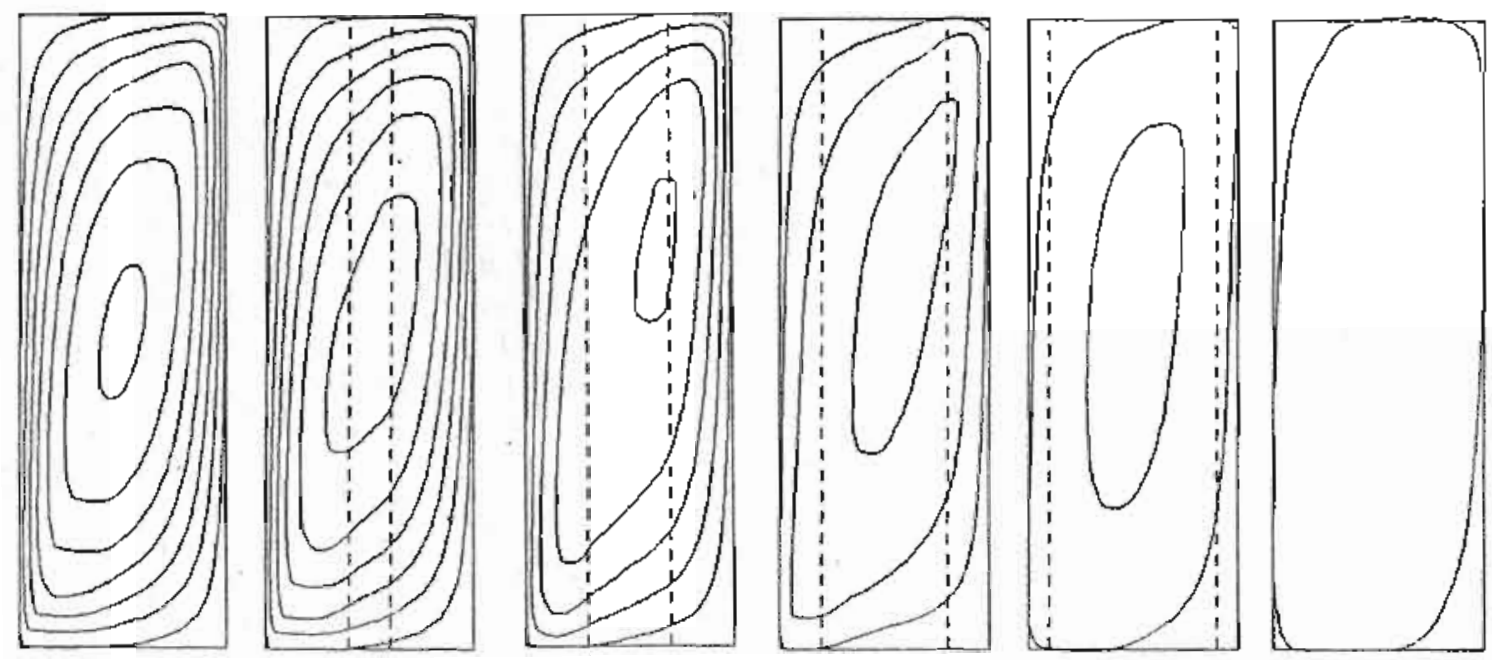

Fig. 8 Streamlines for $A=3, K_{r}=0.2$ and $R a=400$

$$
\left(\Psi_{1}=5, \Delta \Psi=5\right)
$$
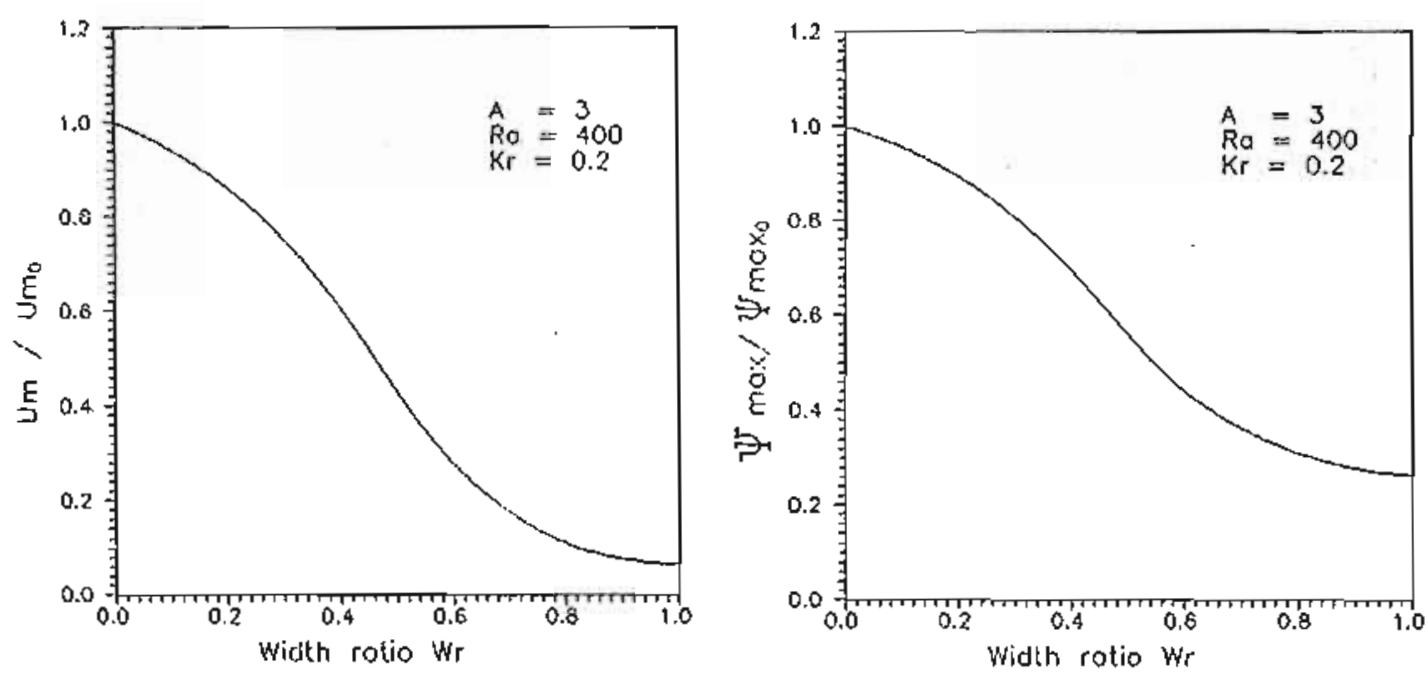

Fig. 9 Variation of the nondimensional average velocity and Streamfunction with the width ratio of the inner sublayer for $A=3, K_{r}=0.2$ and $\mathrm{Ra}=400$ 
M. 108 Dr. M.S.EL KADY
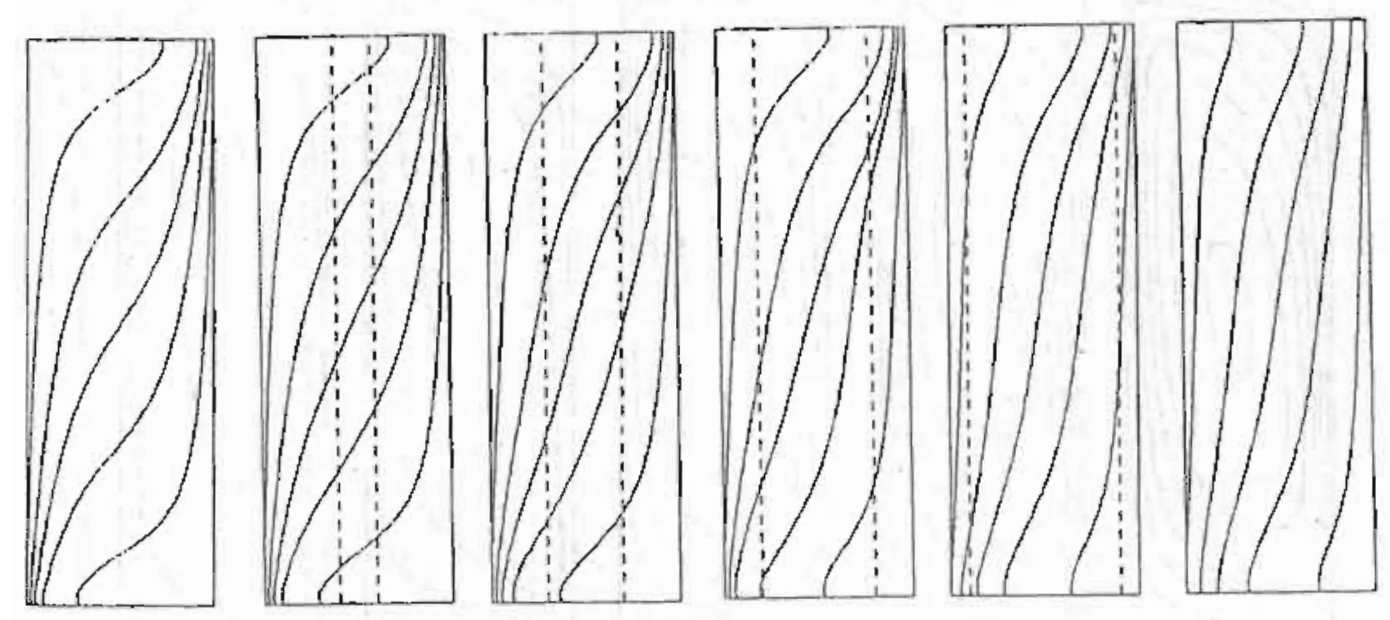

Fig. 10 isotherms for $A=3, K_{r}=0.2$ and $R a=400$

$$
\left(\theta_{1}=0.1, \Delta \theta=0.2\right)
$$
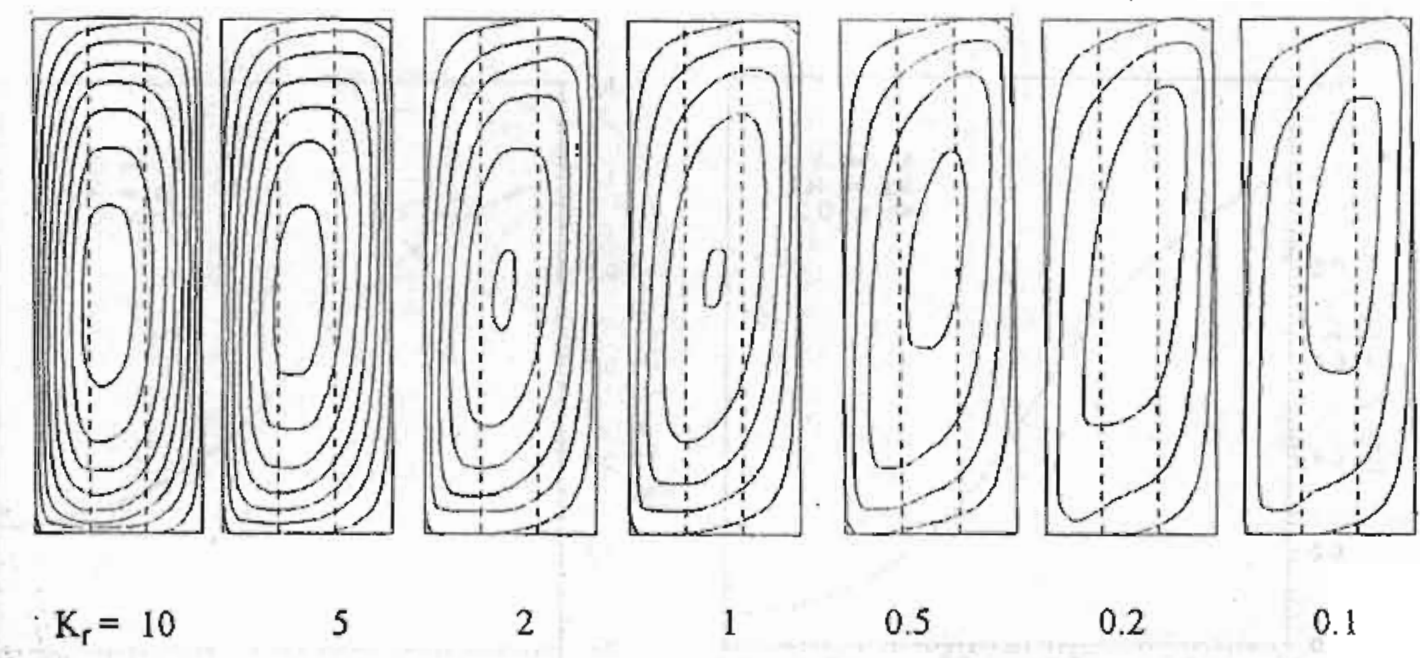

$K_{\mathrm{r}}=10$

5

2

1

0.5

0.2

0.1

Fig. 11 Streamlines for $A=3, W_{r}=0.333$ and $R a=250$ $\left(\Psi_{1}=5, \Delta \Psi=5\right)$ 

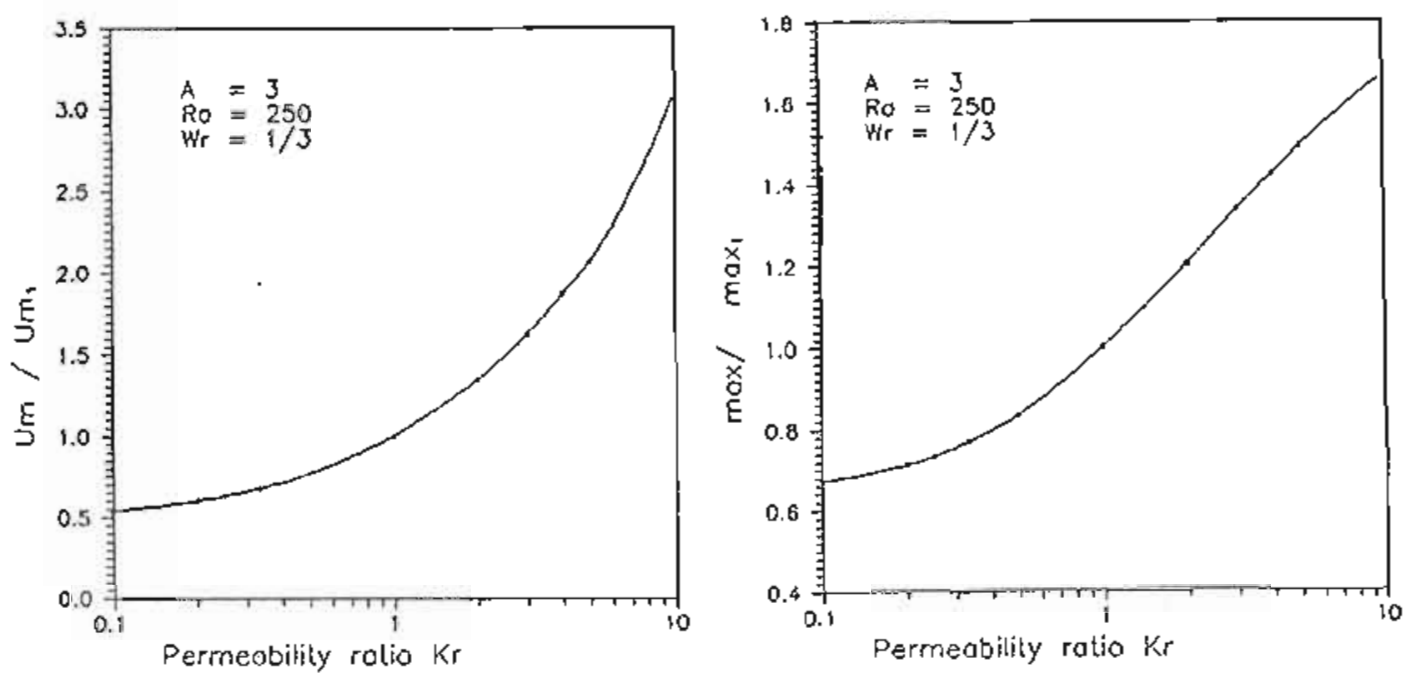

Fig. 12 Varialion of the nondimensional average velocity and Streamfunction with the permeability ratio of the inner sublayer for $A=3, W_{r}=1 / 3$ and $R a=250$
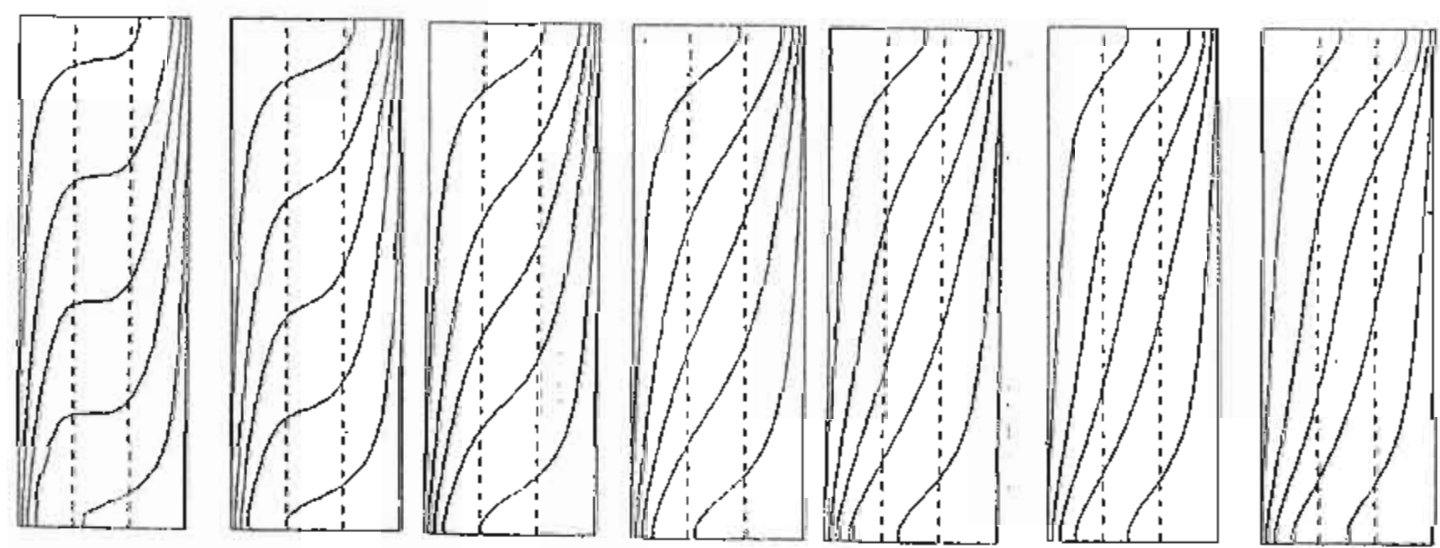

$$
\mathrm{K}_{\mathrm{r}}=10
$$

$$
5
$$

2

1

0.5

0.2

0.1

Fig. 13 Isotherms for $A=3, W_{r}=0.333$ and $R a=250$

$$
\left(\theta_{1}=0.1, \Delta \theta=0.2\right)
$$

